\title{
NO EVIDENCE FOR INHIBITORY DEFICITS OR ALTERED REWARD PROCESSING IN
}

\section{ADHD: DATA FROM A NEW INTEGRATED MONETARY INCENTIVE DELAY}

\section{GO/NO-GO TASK}

\author{
Ellen Demurie ${ }^{1}$, Herbert Roeyers ${ }^{1}$, Jan R. Wiersema ${ }^{1}$, \& Edmund Sonuga-Barke ${ }^{1,2}$ \\ ${ }^{1}$ Department of Experimental Clinical and Health Psychology, Ghent University, Belgium \\ ${ }^{2}$ Developmental Brain-Behaviour Laboratory, University of Southampton, UK
}

Note: This is an uncorrected version of an author's manuscript accepted for publication. Copyediting, typesetting, and review of the resulting proofs will be undertaken on this manuscript before final publication. During production and pre-press, errors may be discovered that could affect the content.

Correspondence to Ellen Demurie, Ghent University, Department of Experimental Clinical and Health Psychology, Henri Dunantlaan 2, B-9000 Ghent, Belgium. Telephone: +32(0)9-2649411. Email: Ellen.Demurie@UGent.be.

Acknowledgements: We would like to thank all the participating children and adolescents, their parents and teachers. This research was supported by a grant from the Fund for Scientific Research - Flanders (Belgium) (FWO), awarded to the first author. 


\begin{abstract}
Objective: Cognitive and motivational factors differentially affect individuals with mental health problems such as attention-deficit/hyperactivity disorder (ADHD). Here we introduce a new task to disentangle the relative contribution of inhibitory control and reward anticipation on task performance in children with ADHD and/or autism spectrum disorders (ASD). Methods: Typically developing children, children with ADHD, ASD or both disorders worked during separate sessions for monetary or social rewards in go/no-go tasks with varying inhibitory load levels. Participants also completed a monetary temporal discounting (TD) task. Results: As predicted task performance was sensitive to both the effects of anticipated reward amount and inhibitory load. Reward amount had different effects depending on inhibitory load level. TD correlated with inhibitory control in the ADHD group. Discussion: The integration of the monetary incentive delay and go/no-go paradigms was successful. Surprisingly there was no evidence of inhibitory control deficits or altered reward anticipation in the clinical groups.
\end{abstract}

Keywords: Attention-Deficit/Hyperactivity Disorder, Autism Spectrum Disorder, Reward processing, Response inhibition 


\section{INTRODUCTION}

Consistent with a number of theories (e.g., Barkley, 1997; Nigg, 2001) studies with both children and adults provide evidence for a link between attention-deficit/hyperactivity disorder (ADHD) and inhibitory dysfunction (Boonstra, Kooij, Oosterlaan, Sergeant, \& Buitelaar, 2010; Lijffijt, Kenemans, Verbaten, \& van Engeland, 2005; Oosterlaan, Logan, \& Sergeant, 1998). Barkley (1997) argued that a deficit in behavioural response inhibition is the core deficit in ADHD and is specifically related to the disorder. Others see deficient inhibitory performance in ADHD rather as a consequence of a non-optimal energetic state (Sergeant, Oosterlaan, \& Van der Meere, 1999). However, there are also studies that do not find these inhibitory problems in ADHD (Daugherty, Quay, \& Ramos, 1993; Jennings, van der Molen, Pelham, Debski, \& Hoza, 1997; Raymaekers, Antrop, van der Meere, Wiersema, \& Roeyers, 2007; Scheres, Oosterlaan, \& Sergeant, 2001). One possible explanation is that variations in cognitive and motivational characteristics of tasks can impact on ADHD children's performance (Johnson, Wiersema, \& Kuntsi, 2009; Luman, Oosterlaan, \& Sergeant, 2005; Scheres et al., 2001). Worse task performance in ADHD might be due to cognitive factors other than inhibitory load (Banaschewski et al., 2004; Lijffijt et al., 2005; Rommelse et al., 2007). This has been investigated by manipulating inhibitory load within the same task (e.g., Van De Voorde, Roeyers, Verté, \& Wiersema, 2010) by altering the ratio of no-go versus go trials (Ciesielski, Harris, \& Cofer, 2004). In general, participants make more commission errors in a high (e.g., 75-25 go/no-go) versus low (e.g., 25-75 go/no-go) inhibitory task condition (Braver, Barch, Gray, Molfese, \& Snyder, 2001; Ciesielski et al., 2004). Van De Voorde et al. (2010) found increased inaccuracy in ADHD in both a high (8020 go/no-go) and low inhibitory load condition (50-50 go/no-go). However, this group effect was not related to inhibitory load (as reflected in the absence of a group by inhibitory load interaction effect), indicating that inaccuracy in ADHD may be due to other factors, which is 
inconsistent with the inhibitory deficit view. Rommelse et al. (2007) concluded that poor performance of children with ADHD on inhibition measures may result from deficiencies in lower order cognitive processes and not only from executive functioning deficits.

Furthermore besides the impact of task characteristics, some have argued that deficits seen in ADHD on inhibitory control tasks may be due not to problems with dealing with inhibitory tasks but rather to a general lack of motivation, which can be addressed by adding rewards. There is some evidence to support this: children with ADHD can perform well on inhibition tasks when properly motivated. For instance, Slusarek, Velling, Bunk, and Eggers (2001) found that ADHD children's inhibitory performance on a stop-signal task was only worse than typically developing controls under a low reward condition. However, when given high levels of reward, children with ADHD performed as well as controls. Also, Konrad, Gauggel, Manz, and Schöll (2000) found that inhibitory control deficits in children with ADHD during a Stop Signal Task could be normalised through non-social incentives. Kohls, Herpertz-Dahlmann, and Konrad (2009) found that both money and social reward (smiling faces) improved inhibitory performance of children with ADHD and controls, although monetary incentives had a substantially stronger effect than social incentives. For the ADHD sample however, social rewards had a stronger effect on improving response inhibition compared to the control group, an effect interpreted as consistent with a hypersensitivity to social rewards in children with ADHD. However, there are also negative results. Oosterlaan and Sergeant (1998) found that impairments in children with ADHD were present despite the delivery of rewards or escape from response cost for correct inhibitions. They concluded that ADHD involves a fundamental deficit in response inhibition. However, Slusarek et al. (2001) argued that Oosterlaan and Sergeant (1998) did not use different intensities of the incentives and that their design was not able to adequately measure differences in motivational dispositions. Despite the arguments of Slusarek et al. (2001) some more recent studies using 
either a stop task (Shanahan, Pennington, \& Willcutt, 2008) or go/no-go task (Desman, Petermann, \& Hampel, 2008; Wodka et al., 2007) have also failed to find motivational modulation of inhibition in ADHD.

When interpreting these inconsistent results, one should take into account the finding that individuals with ADHD process cues of upcoming rewards differently from typically developing peers (e.g., Haenlein \& Caul, 1987; Sagvolden, Johansen, Aase, \& Russell, 2005; Sonuga-Barke \& Fairchild, 2012). For instance, children with ADHD have an unusually strong preference for immediate reward, even if they have the opportunity to choose a larger delayed reward. This characteristic plays a central role in the delay aversion theory of Sonuga-Barke, Taylor, Sembi, and Smith (1992) and the steeper and shorter delay-ofgratification gradient as hypothesized by Sagvolden, Aase, Zeiner, and Berger (1998). A second line of motivational research in ADHD shows that reward and response cost have a positive effect on task performance and motivation of children (controls and ADHD), with sometimes a normalization of the performance of children with ADHD (Luman et al., 2005). The positive effect of reinforcement on cognitive skills seems to be larger for children with ADHD than for typically developing controls (Luman et al., 2005; Luman, Tripp, \& Scheres, 2010). However other researchers suggest a hyposensitivity to reward cues in ADHD (Haenlein \& Caul, 1987). Evidence from functional magnetic resonance imaging (fMRI) studies is more consistent, showing a reduced activation in the ventral striatum of adolescents and adults with ADHD during reward anticipation (Scheres, Milham, Knutson, \& Castellanos, 2007; Ströhle et al., 2008).

In trying to reconcile the contradictory results of the impact of these two factors (inhibitory load and reward processing) it becomes essential to be able to disentangle their specific effects from one another within the same task. In this paper we introduce a new task designed to study the interaction between the effects of inhibitory control and reward 
anticipation on task performance. Most studies of reward and response inhibition in ADHD focus on the receipt of a specific reward, but do not specifically investigate the potential impact of reward anticipation. The monetary incentive delay (MID) task of Knutson, Fong, Adams, Varner, and Hommer (2001) was designed to distinguish the phase of reward anticipation and receipt of the outcome for use in fMRI research. A cue at the beginning of each trial denotes the magnitude of the reward that can be received at the end of the trial after a correct response. The MID task can also be used to tap behavioural effects (Demurie, Roeyers, Baeyens, \& Sonuga-Barke, 2011). Spreckelmeyer et al. (2009) and Rademacher et al. (2010) adapted the original MID task by adding different monetary and social reward intensities. Three types of smiling faces with increasing happiness intensity were used as social rewards. Reaction times (RT) decreased with increasing level of anticipated reward (Rademacher et al., 2010; Spreckelmeyer et al., 2009). A similar task design was used by Demurie et al. (2011). They operationalized social reward as a pictogram of a person approving the performance of another person with thumbs up and a compliment in a text balloon combined with points. Similar to Rademacher et al. (2010) they found a linear effect of monetary and social reward intensity on the accuracy and RT of children with ADHD, autism spectrum disorder (ASD) and typically developing controls. Both clinical groups, but not controls, responded faster to monetary than social rewards. There was thus no evidence for a hypersensitivity to social rewards in ADHD (Demurie et al., 2011).

In the current study the MID task, as the initial concept, was adapted and integrated into a go/no-go paradigm in which the frequency of go and no-go trials was varied to produce settings with high (i.e., a low proportion of no-go trials) and low inhibitory load (i.e., a low proportion of go trials). Several aspects of the task design are noteworthy. First, in many of the aforementioned studies on motivational effects on response inhibition, the stop task (Logan, 1994) was used as the inhibition measure. However, researchers have expressed 
reservations with regard to the appropriateness of the stop task as a measure of inhibition in ADHD. Stop-signal reaction time differences are suggested to reflect a more generalized deficit in attention or cognitive processing in ADHD, rather than behavioural inhibition problems (Alderson, Rapport, \& Kofler, 2007). Therefore, we wanted to replicate prior findings with a go/no-go task. Second, the effects of monetary and social reward anticipation were compared. Finally, both correct go- and no-go-responses were rewarded to reduce the chance that differences between individuals in terms of the strategies adopted could explain the results. Scheres et al. (2001) found that reward improved overall task performance of both children with ADHD and typically developing children during a stop task where reward was only given after successful inhibition, and not after fast response execution. However, children with ADHD slowed down more in the reward condition than in the non-reward condition compared to controls. This has been interpreted as a strategy effect and thus a stronger tendency to seek reward in children with ADHD.

Usually reward processing and inhibitory abilities are investigated in both a sample of children with ADHD and a control group of typically developing children (Luman et al., 2005). However, children with ASD also show problems with response inhibition (Bishop \& Norbury, 2005; Geurts, Verté, Oosterlaan, Roeyers, \& Sergeant, 2004; Verté, Geurts, Roeyers, Oosterlaan, \& Sergeant, 2005) and differences in reward processing (Dawson, Osterling, Rinaldi, Carver, \& McPartland, 2001), mainly with regard to social rewards (Chevallier, Kohls, Troiani, Brodkin, \& Schultz, 2012; Demurie et al., 2011; Garretson, Fein \& Waterhouse, 1990; Geurts, Luman, \& van Meel, 2008; Schultz, 2005; Scott-Van Zeeland et al., 2010; but see Dichter, Richey, Rittenberg, Sabatino, \& Bodfish, 2012; Kohls et al., 2012). In order to examine to what extent abnormal reward processing and inhibition problems in ADHD are disorder-specific, this study also included a group of children with ASD and a group of children with a comorbid diagnosis of ADHD and ASD. 
The main goal of this study was to develop a new paradigm that integrated the MID task with a go/no-go paradigm. We had a number of research questions. First we tested whether the task could demonstrate the effects of reward anticipation, inhibitory demands and their interaction. We predicted (1) a main effect of inhibitory load, with more commission errors in the high inhibitory load condition, (2) a main effect of reward amount, with better performance with increasing rewards and (3) an interaction between reward amount and inhibitory load - with the effects of reward amount being most pronounced under the low inhibitory load conditions (Leotti \& Wager, 2010). Second, we looked at the effects of reward type (money versus social rewards). We predicted stronger effects of monetary as opposed to social rewards (Kohls et al., 2009). Third, we explored the effects of psychopathology on the effects of the relevant task parameters. To do this we compared a group of children with ADHD with a typically developing control group, a group of children with ASD and one with both ADHD and ASD. On the basis of prior work we predicted (1) worse inhibitory performance in all clinical groups especially in the high inhibitory load condition and (2) a greater effect of reward amount on the children with ADHD. Since this is the first study to investigate the effects of reward on inhibition in an ASD group and a comorbid group (ADHD+ASD), we had no clear predictions for these groups. (3) In line with Demurie et al. (2011) we expected a stronger effect of monetary rewards in the clinical groups. Our final aim addressed Mischel et al.'s (2011) suggestion of an interrelation between executive control and delay-of-gratification. However, Solanto et al. (2001) and Karalunas and Huang-Pollock (2011) found that deficits in inhibition (as measured by stop-signal RT) were uncorrelated to the tendency to choose a smaller immediate reward rather than a larger delayed reward, which suggests that inhibitory deficits and delay aversion are independent characteristics. Moreover both characteristics were strongly and independently associated with ADHD (Solanto et al., 2001). In their studies response inhibition was measured with the Stop Signal Task (Logan, 
1994) and delay aversion with the Choice Delay Task (CDT; Sonuga-Barke et al., 1992). The CDT uses a fixed-choice design that does not give insight into how trade-offs are made between reward magnitude and delay duration before reward delivery. One of the main advantages of temporal discounting (TD) tasks is that they allow for measuring this trade-off (Scheres et al., 2006). The subjects in the current study performed a monetary TD task and different go/no-go tasks, which gave us the opportunity to investigate the association between TD and inhibitory performance. In line with the suggestion of Mischel et al. (2011) we expected significant associations between TD and number of commission errors in the go/nogo tasks.

\section{METHODS}

\section{Participants}

41 typically developing children, 34 children with ADHD, 36 with ASD and 16 with a comorbid diagnosis of ADHD and ASD took part. Each (8-16 years old) had an IQ of 80 or more, measured on 4 subscales of the Wechsler Intelligence Scale for Children $-3^{\text {rd }}$ edition (Wechsler, 1991; similarities, vocabulary, block design and picture arrangement). The sum of these subtest scores gives a reliable estimate of the FSIQ (Grégoire, 2005). Groups did not differ in age $(\mathrm{p}=.86)$, IQ $(\mathrm{p}=.12)$, sex ratio $(\mathrm{p}=.22$; see Table 1$)$ or distribution over the five social classes as measured by the Hollingshead Index $\left(\gamma^{2}(9)=8.63, p=.47\right)$.

Control children were recruited trough letters to local elementary and secondary schools and targeted advertisements in magazines. All control children were nominated by their parents as not displaying behaviour problems. The participants with ADHD and/or ASD were all outpatients, recruited through rehabilitation centres, special school services, and other agencies specialized in the care of children with developmental disorders. All had previously been diagnosed with ADHD and/or ASD by a multidisciplinary team according to DSM-IVTR (American Psychiatric Association, 2000) criteria. Clinical diagnoses were confirmed 
with a validated measure: the Autism Diagnostic Interview, Revised (ADI-R; Lord, Rutter, \& Lecouteur, 1994), Autism Diagnostic Observational Schedule, Generic (ADOS-G; Lord et al., 2004) or Childhood Autism Rating Scale (CARS; Schopler, Reichler, Devellis, \& Daly, 1980) for ASD. The ADHD diagnosis was verified using the disruptive behaviour module (ADHD, oppositional defiant disorder, conduct disorder) of the Diagnostic Interview Scale for Children for DSM-IV (Shaffer, Fisher, Lucas, Dulcan, \& Schwab-Stone, 2000). In the ADHD sample, 10 participants met the criteria for the inattentive subtype, 4 for the hyperactive subtype and 20 for the combined subtype. Furthermore, 16 participants also fulfilled the diagnostic criteria of ODD, but none of the participants with ADHD had comorbid CD. In the comorbid sample, 7 participants met the criteria for the inattentive subtype, 2 for the hyperactive subtype and 7 for the combined subtype. 6 participants with a comorbid diagnosis also showed the diagnostic criteria of ODD, but none had comorbid CD. Participants on stimulant medication were asked to discontinue their medication at least 24 hours prior to testing. 22 participants with ADHD were medicated with methylphenidate (MPH), 4 participants with ASD and 11 participants with ADHD and ASD.

Parents and teachers completed questionnaires to obtain a broad view of the child's overall functioning and possible comorbid symptoms. Parents completed the Disruptive Behavior Disorder rating scale (DBDRS; Pelham, Gnagy, Greenslade, \& Milich, 1992) and Social Communication Questionnaire (SCQ; Rutter, Bailey, \& Lord, 2003). Teachers completed the DBDRS.

\section{Monetary and Social Incentive Delay (MID and SID) go/no-go tasks}

The tasks were based on Knutson's MID task (Knutson et al., 2001). A cue at the start of each trial indicated the motivational consequences of good performance. In the current version an empty circle indicated no reward, one with one line a medium reward and one with three lines a high reward for good performance. In the MID/SID go/no-go tasks children were 
told that they could earn money (MID) or points (SID) during the task. The investigator told them that they would see a cue at the start of each trial that would show how much they could win during that specific trial. Furthermore, the meaning of the cues was explained and the children were told how to react to the target stimuli. It was stressed that they had to react as quickly as possible, but also had to try to be as accurate as possible. These instructions were illustrated with pictures of the cues and targets and presented in four different instruction windows. The instructions were then followed by ten target trials.

The classic MID task was adapted in three ways. First of all, the simple target detection task typically used was replaced by a rewarded go/no-go task. The go/no-go paradigm is one of the most frequently used response inhibition measures (Simmonds, Pekar, \& Mostofsky, 2008). In this paradigm either a go (square) or a no-go stimulus (triangle) is presented on the screen in each trial. Children have to press a button when they see a go-stimulus but avoid responding when they see a no-go stimulus. By adding this go/no-go aspect to the MID task, we wanted to measure the interaction between reward anticipation and response inhibition processes. To do this we compared conditions with different inhibitory load levels: a 75-25 go/no-go condition, which loaded high on inhibitory demands, and a 25-75 go/no-go condition with low inhibitory demands. In the $75-25$ condition, there were 81 go and 27 no-go trials. In the 25-75 condition, there were 27 go-trials and 81 no-go trials. Second, in the original MID task difficulty was set such that participants would succeed on approximately $66 \%$ of all trials, based on RTs collected during a practice session. In our adaptation performance level was not individually tailored, as we wanted to be able to investigate group differences in both RT and task accuracy as well as in total amount of gain. Third, we compared the effects of monetary and social rewards. In the monetary (MID) conditions, participants could win eurocents for a fast and correct reaction. A cue presented at the start of each trial signalled the size of the reward to be delivered. Three reward intensities were 
possible: no reward ( 0 eurocent), medium reward or high reward. Rewards for correct inhibitions and correct go-responses were in proportion with the go/no-go rate, thus the $25 \%$ reactions were rewarded 3 times as high as the $75 \%$ reactions. By doing so, alternative strategies, such as always or never pressing the button, could be discouraged. A fast and/or correct reaction was rewarded 4 (75\% reaction) or 12 eurocents (25\% reaction) after a medium reward cue and 10 or 30 eurocents respectively after a high reward cue (see Figure 1 for an overview of the meaning of the cues in the 75-25 monetary task). All participants correctly believed that they would receive the obtained amount of money at the end of the testing. During the social (SID) conditions, reward was operationalized as $0,4,12,10$ or 30 points, in combination with a pictogram of an interaction between two persons. One person in the pictogram approves the performance of the other person with thumbs up and a compliment in a text balloon. The compliments were different in each block, to avoid habituation. Examples are 'Ok' as a 0 points compliment, 'Well done' for a medium reward and 'You're a champion!' for a high reward. Figure 2 shows an example trial of each task.

Each child performed under two conditions (high versus low inhibitory load), so that there were in total 4 conditions: monetary-high inhibition load (MID-High), monetary-low inhibition load (MID-Low), social-high inhibition load (SID-High) and social-low inhibition load (SID-Low). In each condition there were 10 practice trials and three blocks of 36,6 sec trials, yielding a total of 118 trials. During each trial, participants saw one of three cue shapes (500 msec), followed by a black screen (pre-target delay, random variable duration: 2000$2500 \mathrm{msec}$ ) and the target stimulus (triangle or square, $260 \mathrm{msec}$ ). During go-trials participants had to react within a $500 \mathrm{msec}$ response window to be rewarded. During no-go trials, a button press during the post-target delay was considered incorrect. After the posttarget delay (variable duration dependent of pre-target delay duration: 1000-1500 msec), a feedback screen (1740 msec) notified participants of whether they had won money (monetary 
task) or points (social task) during that trial and indicated their cumulative total at that point. The order of the four conditions was counterbalanced among the participants.

\section{Monetary temporal discounting task}

Participants also performed a computerized monetary TD task. They were instructed to make repeated choices between (hypothetical) small variable rewards delivered immediately and a large constant reward (30€) delivered after a variable delay (now, tomorrow, the day after tomorrow, 1 week, 2 weeks) (see Figure 3). Reward magnitudes were $0 €, 5 €, 10 €, 20 €$ and $30 €$, visualized as euro notes. Each small immediate reward was paired four times with every delay for the large reward, resulting in a total of 100 choice trials. Trials were administered in the same pseudo-random order to all participants. Left or right position of the delayed reward was balanced over trials.

We have chosen to use hypothetical rewards and delays in this study, thus the children did not actually receive the rewards, neither did they experience real delays, but they had to imagine that they would receive these rewards after a given delay. Results from TD tasks with real rewards and delay intervals have previously been shown to be highly correlated with those of hypothetical TD tasks (Johnson \& Bickel, 2002; Scheres, Sumiya, \& Thoeny, 2010), which suggests that there is congruency between what participants believe they would choose and what they actually choose. Both real and hypothetical TD tasks are thus reliable measures of TD and give similar results.

\section{Data preprocessing of the TD task}

Preprocessing of TD data was based on procedures described by Critchfield and Kollins (2001) and Scheres et al. (2006) and has been explained into detail in Demurie, Roeyers, Baeyens, and Sonuga-Barke (2012). First, subjective values of the delayed reward were determined for each delay by two independent raters. The subjective value of the delayed reward was estimated by averaging the switch points in the ascending and descending choice 
sequences (Critchfield \& Kollins, 2001). These switch points are defined as the magnitudes of the small immediate reward for which the participant shows indifference in a choice against the large delayed reward (Scheres et al., 2006). The second step in the preprocessing of the data was to calculate the area under the curve (AUC) for the TD functions according to the procedure described by Myerson, Green, and Warusawitharana (2001). The steeper the discounting function, the smaller the AUC will be.

\section{Procedure}

Children and their parents were invited to the Faculty of Psychology and Educational Sciences. All participants and parents signed an informed consent form prior to testing. At the beginning of the test session we verified whether participants on methylphenidate were really off medication for at least 24 hours by asking when they had taken their last dose of methylphenidate. The study was approved by the Ethical Committee of the Faculty of Psychology and Educational Sciences of Ghent University.

\section{Dependent measures and data analysis}

The AUC reflects the steepness of the TD slopes. Percentage of commission errors (pressing the button after a no-go target) has been used as a measure of response inhibition, with more errors reflecting more deficient response inhibition. Other dependent measures were percentage of omission errors (not pressing the button after a go target), mean reaction time of go-responses (RT) and within-subject variability, operationalized as within-subject standard deviation of RTs of go-responses (SD-RT). Responses faster than $150 \mathrm{msec}$ were considered to be premature responses and excluded from the analyses.

A 4 ('Group': controls vs. ADHD vs. ASD vs. ADHD+ASD) x 2 ('Inhibitory load': high inhibitory load - 75/25 go/no-go task vs. low inhibitory load - 25/75 go/no-go task) x 2 ('Reward type': monetary vs. social reward) x 3 ('Reward amount': no reward vs. medium reward vs. high reward) design was used in repeated measures analyses. To answer the 
research questions, the focus was on the main and two-way interaction effects. Furthermore, correlations between AUC and number of commission errors have been calculated.

\section{RESULTS}

\section{Are the manipulations of inhibitory load and reward amount successful?}

Was there a main effect of inhibitory load? There were main effects of inhibitory load on all dependent variables. A higher percentage of commission errors was seen in the high inhibitory load condition $(\mathrm{F}(1,122)=641.87, \mathrm{p}<.001)$. Omission errors on the other hand were more frequent in the low inhibitory load condition $(\mathrm{F}(1,122)=133.62, \mathrm{p}<.001)$. Participants responded faster $(\mathrm{F}(1,116)=119.70, \mathrm{p}<.001)$, but with more intra-subject variability $(\mathrm{F}(1,98)=16.58, \mathrm{p}<.001)$ in the high inhibitory load condition.

Was there a main effect of reward amount? Main effects of reward amount were found for all go-response related measures, but not for commission errors. For omission errors $(\mathrm{F}(2,121)=60.91, \mathrm{p}<.001)$ and $\mathrm{RT}$ of correct go-responses $(\mathrm{F}(2,115)=30.89, \mathrm{p}<.001)$ performance improved for each increase in anticipated reward (Om/RT no reward $>$ Om/RT medium reward $>$ Om/RT high reward). Furthermore, participants' RTs became less variable in the high reward trials compared to the medium reward trials $(F(2,97)=4.43, \mathrm{p}<.05)$.

Was there an interaction between inhibitory load and reward amount? For commission errors, an interaction between inhibitory load and reward amount $(\mathrm{F}(2,121)=$ $8.68, \mathrm{p}<.001$ ) was found (see Figure 4 ). In the high inhibitory load condition, there was an effect of reward amount $(\mathrm{F}(2,122)=4.06, \mathrm{p}<.05)$, showing a lower percentage of commission errors in the high reward trials compared to the medium reward trials (comm 12 $>$ comm 30; $<<.05)$. In the low inhibitory load condition, the effect of reward intensity $(\mathrm{F}(2,121)=8.25, \mathrm{p}<.001)$ showed the reversed pattern: surprisingly participants made most errors in the high reward condition $($ comm $0=$ comm $4<$ comm $10 ; \mathrm{p}=.34, \mathrm{p}<.001$ and $\mathrm{p}<$ .01 respectively). 
For omission errors the interaction between inhibitory load and reward amount $(\mathrm{F}(2,121)$ $=12.18, \mathrm{p}<.001$, see Figure 5) pointed out that greater effects of reward amount could be found in the low compared to the high inhibitory load conditions $(F(1,125)=4.77, p<.05$ for medium versus no reward; $F(1,125)=27.85, \mathrm{p}<.001$ for high versus no reward; $F(1,125)=$ $11.56, \mathrm{p}<.01$ for high versus medium reward).

For RT of correct go-responses, the interaction between inhibitory load and reward amount $(\mathrm{F}(2,115)=15.92, \mathrm{p}<.001)$ showed that there was a greater decline in RTs with increasing reward in the low inhibitory load condition (RT $0>\mathrm{RT} 12>\mathrm{RT} 30$ ) compared to the high inhibitory load condition (RT $0=$ RT $4>$ RT 10). Finally for SD-RT, the interaction between inhibitory load and reward amount was not significant $(F(2,97)=2.48, \mathrm{p}=.09)$.

\section{Is there a different effect of monetary versus social reward anticipation?}

Reward type effects were only found for the go-response variables. For omission errors a main effect of reward type $(\mathrm{F}(1,122)=25.81, \mathrm{p}<.001)$ was found, with a lower percentage of omission errors in the monetary conditions. An interaction between reward type and amount $(\mathrm{F}(2,121)=3.53, \mathrm{p}<.05)$ showed that this effect could only be seen in the rewarded trials. Furthermore, an interaction between inhibitory load and reward type $(\mathrm{F}(1,122)=3.75, \mathrm{p}=$ $.055)$ demonstrated that greater differences between monetary and social rewards $(\mathrm{F}(1,125)=$ $3.97, \mathrm{p}<.05)$ could be found in the low compared to the high inhibitory load conditions. Furthermore, RT of correct go-responses were faster when money could be won $(\mathrm{F}(1,116)=$ $4.20, \mathrm{p}<.05)$. Finally, an interaction effect between inhibitory load and reward type $(\mathrm{F}(1,98)$ $=5.65, \mathrm{p}<.05)$ was found for SD-RT: only in the high inhibitory load condition less variable RTs were observed when money compared to social rewards could be won $(\mathrm{p}<.01)$.

\section{What are the effects of psychopathology on the effects of the relevant task parameters?}


Was there a group difference in overall task performance? No group differences in percentage of commission errors $(\mathrm{F}(3,122)=1.32, \mathrm{p}=.27)$, $\mathrm{RT}(\mathrm{F}(3,116)=.41, \mathrm{p}=.75)$ and SD-RT of correct go-responses $(\mathrm{F}(3,98)=1.60, \mathrm{p}=.19)$ were found. For omission errors $(\mathrm{F}(3,122)=3.36, \mathrm{p}<.05)$ on the other hand, a difference between the controls and the comorbid group could be observed, with the latter ones making more errors.

Were there group differences in the impact of anticipated rewards? An interaction between reward amount and group $(\mathrm{F}(6,242)=2.52, \mathrm{p}<.05$; see Figure 6$)$ for omission errors demonstrated that a group difference could be observed in the no-reward trials only: participants with a combined diagnosis of ADHD+ASD had a higher percentage of omission errors than controls and participants with ASD (with the ADHD participants in between). Performance of the participants with ADHD+ASD thus normalized in the rewarded trials. For the other variables, no interactions between reward amount and group were found.

Was there a different effect of monetary versus social reward anticipation in the participating groups? No interaction effects between group and reward type were found for any of the dependent variables.

\section{Is there an association between TD and inhibitory performance?}

Correlations between the AUC for money and the percentage of commission errors in the go/no-go tasks were calculated for the four participating groups. Only in the ADHD sample, significant correlations between monetary AUC on the one hand and the percentage of commission errors in the MID-High condition $(\mathrm{r}(32)=-.41, \mathrm{p}<.05)$ and the percentage of commission errors in the SID-High condition $(\mathrm{r}(32)=-.52, \mathrm{p}<.01)$ on the other hand have been found. These correlations remained significant if IQ was controlled for.

\section{DisCUSSION}


In the current study, we successfully adapted the MID task (Knutson et al., 2001) to investigate the interaction between reward anticipation and inhibitory control. We did this by integrating this task into a go/no-go paradigm to compare two levels of inhibitory load and three amounts of reward. We were able to formulate answers to the four sets of predictions. The first set of predictions related to the task manipulations, which were successful: we observed the predicted effects of inhibitory load and reward amount, as well as interactions between both factors. This interaction showed that anticipated rewards differentially affected task performance in the two inhibition conditions. With regard to commission errors, we found a positive effect of reward anticipation on inhibitory performance in the high inhibitory load condition: fewer errors were made in the high-reward trials compared to the medium reward trials. Inhibitory performance can thus be influenced by reward anticipation, irrespective of group. These results are in line with previous reports of improved inhibitory performance due to reward (e.g., Kohls et al., 2009; Luman et al., 2005). Surprisingly, in the low inhibitory load condition the percentage of commission errors increased in the highest reward condition. This was possibly due to the fact that the reward for correct go-trials was three times higher than for no-go-trials (30 versus 10 points/eurocents) in the low inhibitory load condition which made 'pressing the button' a more prepotent response in the highreward trials, although this was not expected given the low frequency of go-trials $(25 \%)$. The cue for a high reward seemed to be a salient cue that made suppressing the go-response harder in these trials. Thus our effort to avoid the strategy of 'always pressing the button' in the high inhibitory load condition seems to have had the opposite effect in the low inhibitory load condition. Another possible explanation is that our manipulation evoked a response bias towards or response strategy of always reacting to the high reward predicting cues (to maximize the chance of winning the high go-related reward). Unequal distribution of rewards between two types of correct response produces a systematic preference for the response 
producing the larger reward (Tripp \& Alsop, 1999). Although the overall size of reward was equal for the go and no-go responses, it is possible that participants saw the reward amounts as imbalanced, causing a response bias for the go-response. In any case, with regard to commission errors our results clearly suggest that the effects of reward anticipation on performance are sensitive to variation in inhibitory load. For omission errors and RT of correct go-responses, we found that the effects of anticipated rewards were stronger in the low compared to the high inhibitory load condition. One may argue that there was more room for improvement in the low inhibitory load condition, but there was no evidence for a ceiling effect in the high inhibitory load condition. Leotti and Wager (2010) also found that performance improvement induced by changes in motivational context were less extreme when inhibitory demands were higher. Thus also for the go-responses differential effects of neuropsychological load could be observed. Kohls et al. (2012) suggested that findings of aberrant reward sensitivity should be considered in the context of the cognitive demands required for a task, since subjective reward value varies inversely with the effort necessary to obtain the reward (Botvinick, Huffstetler, \& McGuire, 2009). Krebs, Boehler, Roberts, Song, and Woldorff (2012) showed that the anticipation of both varying reward magnitudes and varying degrees of task difficulty evoked interacting brain activations in the dopaminergic midbrain and cortico-striatal-thalamic circuits, contributing to the modulation of resource recruitment in order to facilitate task performance when it is particularly worthwhile. In line with this, we found that task demands indeed seem to alter sensitivity to anticipated rewards and consequently the selection of task strategies.

Our second set of predictions related to the type of reward employed. Reward type had an impact on all go-response related measures: omission errors and RT in all four tasks and on SD-RT in the high inhibitory load condition. The results are in line with those of Demurie et al. (2011) and Kohls et al. (2009): although both monetary and social reward improved task 
performance of all participants, performance was better when monetary rewards compared to social rewards could be won. For omission errors, an interaction between reward type and reward intensity was found: the main effect of reward type could only be observed in the rewarded trials. These results emphasize the power of money as a reinforcer, both in daily life and in research.

Our third set of predictions related to the comparison of the effects of reward anticipation and inhibitory load in ADHD and/or ASD. Against our predictions and in contrast to a great deal of the existing literature we found very little evidence for differential effects of reward amount or inhibitory load in these groups. First, we found no differences in the percentage of commission errors (both on rewarded and unrewarded trials) in the ADHD or the ASD groups. Nigg (2001) hypothesized that children with ADHD manifest deficits with effortful or controlled inhibition ('executive inhibition') but that inhibition of behaviours or cognitions associated with reward or punishment ('motivational inhibition') is intact. One possible explanation is that the motivational effects of reward anticipation extended to the nonrewarded trials, normalizing inhibitory performance in the clinical groups throughout the entire task. Given the positive influence of motivational variables on effort allocation (Sanders, 1983; Sergeant et al., 1999), reward anticipation might have elevated the energetic state of the children with ADHD to a more optimal level leading to a better inhibitory performance. On the other hand, others have also previously failed to find evidence for impaired inhibitory performance in ADHD or ASD (Daugherty et al., 1993; Ozonoff \& Jensen, 1999; Raymaekers et al., 2007; Van De Voorde et al., 2010). The current results appear to support theoretical accounts questioning the fundamental and unique causal role of executive function deficits in ADHD and ASD (Sergeant, 2000; Sonuga-Barke et al., 1992). Banaschewski et al. (2004) suggested that the occurrence of inhibition problems in ADHD might depend on which tasks are used to measure inhibition. Furthermore go/no-go tasks do 
not tap all aspects of inhibitory performance (Nigg, 2000). We can thus not exclude the possibility that our go/no-go tasks were not sensitive enough to find group differences in inhibitory performance. Future studies should check the baseline sensitivity of tasks in discriminating children with ADHD and typically developing children. Second, except for the omission errors, there were no differential effects of reward amount on performance across the groups: no interactions between group and reward amount were found for commission errors, RT and SD-RT. The current results are in line with the unexpected findings of Demurie et al. (2011), showing no evidence for the predicted hyposensitivity to changes in reward amount in ADHD. Reward anticipation seems to have a general effect on task performance over and above any specific reward or group effects on cognitive control (Geurts et al., 2008). Barkley (1997) postulated that children with ADHD are not more or less sensitive to rewards, but suggested that their behaviour is more influenced by immediate events and their consequences than by experiences more distal in time. In the current adaptation of the MID task, the participants were rewarded immediately after each correct response. As children with ADHD might perform similar to controls under schedules of continuous and immediate reinforcement (e.g., Barber, Milich, \& Welsh, 1996; Parry \& Douglas, 1983), the chosen reinforcement schedule might be the reason why we did not find any differential reward effects in the ADHD sample. However, for omission errors we did find an interaction between group and reward intensity. Only in the no-reward trials, children with a combined diagnosis of ADHD and ASD made more omission errors compared to controls and children with ASD, but this group difference disappeared in the rewarded trials. Given that omission errors have been linked to attentional problems and lapses of attention during response execution (Bezdjian, Baker, Lozano, \& Raine, 2009), our results suggest that attention problems in children with ADHD+ASD are worse compared to controls and children with ASD when they are not rewarded, but normalize when rewards are expected. 
These attention problems in the comorbid group were not reflected in the inattention scores on the questionnaires rated by parents and teachers, as these were not higher compared to the other clinical groups. Is it possible that parents and teachers of children with a comorbid diagnosis of ADHD and ASD in our sample reinforce these kids more systematically in daily life, as learnt in certain parent training courses? Third, against our expectations, the effects of reward type did not interact with group and we did not find evidence for a smaller effect of social as opposed to monetary rewards in the clinical groups (Chevallier et al., 2012; Demurie et al., 2011).

Finally, with regard to our last prediction, evidence for a link between response inhibition and temporal discounting in the ADHD sample was found, since monetary TD correlated negatively with the percentage of commission errors in both the monetary and social high inhibitory load condition, but not with those of the low inhibitory load condition. The steeper the TD slope, the more commission errors were made, suggesting that poor response inhibition and steep TD indeed are associated. Our results are thus in line with the predictions of Mischel et al. (2011). Previous studies, making use of the stop signal task and CDT, found no correlation between delay aversion and inhibitory deficits in ADHD (Karalunas \& HuangPollock, 2011; Solanto et al., 2001). In the current study inhibition was measured with go/nogo tasks and a hypothetical TD task was used to tap delay averse response style. The use of these tasks may have been decisive for finding the association. However, based on the current results nothing can be concluded with regard to the underlying causality of this association. Future studies might further investigate the strength and causal mechanisms of the possible association between temporal discounting and inhibition.

Some limitations should be acknowledged as well. First, we wanted to vary the inhibitory load of the go/no-go tasks, by using both 75-25 and 25-75 go/no-go tasks. However, if we compared task performance on the two tasks, there were not only more commission errors in 
the high inhibitory load condition, but also less omission errors and faster, but more variable responses. As percentage of commission errors is supposed to reflect a measure of inhibitory difficulties and impulsivity, while omission errors have been linked to attentional problems (Bezdjian et al., 2009), this result suggests that the two tasks might tap different cognitive processes, with the high inhibitory load condition tasks focusing mainly on response inhibition and the low inhibitory load condition also measuring attention and target detection. The design of the low inhibitory load condition is in fact that of an oddball task, where subjects are instructed to generate a response to infrequent targets (Braver et al., 2001). These differences in inhibitory and attentional demands may have confounded our results. Second, the sample size of the comorbid group was rather low, which might have had an impact on the power of the effects. On the other hand, this was the only group where we found a group difference in the percentage of omission errors. Third, some evidence for a link between inhibition and TD in the ADHD sample could be found, since monetary TD correlated negatively with the number of commission errors in the high inhibitory load condition. However, these go/no-go tasks also had a reward processing aspect, which may have had a confounding effect on our results, especially if we take abnormal reward processing in ADHD (Luman et al., 2005) into account. Future research should thus make use of 'pure' go/no-go tasks to investigate the association between TD and inhibition performance on go/no-go tasks.

\section{REFERENCES}

Alderson, R.M., Rapport, M.D., \& Kofler, M.J. (2007). Attention-deficit/hyperactivity disorder and behavioral inhibition: A meta-analytic review of the stop-signal paradigm. Journal of Abnormal Child Psychology, 35(5), 745-758. doi: 10.1007/s10802-007-9131-6

American Psychiatric Association (2000). Diagnostic and Statistical Manual of Mental Disorders (4th edition, text revision). Washington, DC: APA. 
Banaschewski, T., Brandeis, D., Heinrich, H., Albrecht, B., Brunner, E., \& Rothenberger, A. (2004). Questioning inhibitory control as the specific deficit of ADHD - Evidence from brain electrical activity. Journal of Neural Transmission, 111(7), 841-864. doi: 10.1007/s00702-003-0040-8

Barber, M.A., Milich, R., \& Welsh, R. (1996). Effects of reinforcement schedule and task difficulty on the performance of attention deficit hyperactivity disordered and control boys. Journal of Clinical Child Psychology, 25(1), 66-76. doi: 10.1207/s15374424jccp2501_8

Barkley, R.A. (1997). Behavioral inhibition, sustained attention, and executive functions: Constructing a unifying theory of ADHD. Psychological Bulletin, 121(1), 65-94. doi: 10.1037//0033-2909.121.1.65

Bezdjian, S., Baker, L.A., Lozano, D.I., \& Raine, A. (2009). Assessing inattention and impulsivity in children during the go/nogo task. British Journal of Developmental Psychology, 27, 365-383. doi: $10.1348 / 026151008 \times 314919$

Bishop, D.V.M., \& Norbury, C.F. (2005). Executive functions in children with communication impairments, in relation to autistic symptomatology - 2: Response inhibition. Autism, 9(1), 29-43. doi: $10.1177 / 1362361305049028$

Boonstra, A.M., Kooij, J.J.S., Oosterlaan, J., Sergeant, J.A., \& Buitelaar, J.K. (2010). To act or not to act, that's the problem: Primarily inhibition difficulties in adult ADHD. Neuropsychology, 24(2), 209-221. doi: 10.1037/a0017670

Botvinick, M.M., Huffstetler, S., \& McGuire, J.T. (2009). Effort discounting in human nucleus accumbens. Cognitive Affective and Behavioral Neuroscience, 9(1), 16-27. doi: 10.3758/CABN.9.1.16

Braver, T.S., Barch, D.M., Gray, J.R., Molfese, D.L., \& Snyder, A. (2001). Anterior cingulate cortex and response conflict: Effects of frequency, inhibition and errors. Cerebral Cortex, 11(9), 825-836. doi: 10.1093/cercor/11.9.825

Chevallier, C., Kohls, G., Troiani, V., Brodkin, E.S., \& Schultz, R.T. (2012). The social motivation theory of autism. Trends in Cognitive Sciences, 16(4), 231-239. doi: 10.1016/j.tics.2012.02.007

Ciesielski, K.T., Harris, R.J., \& Cofer, L.F. (2004). Posterior brain ERP patterns related to the go/no-go task in children. Psychophysiology, 41(6), 882-892. doi: 10.1111/j.1469-8986.2004.00250.x

Critchfield, T.S., \& Kollins, S.H. (2001). Temporal discounting: Basic research and the analysis of socially important behavior. Journal of Applied Behavior Analysis, 34(1), 101-22. doi: 10.1901/jaba.2001.34-101

Daugherty T.K., Quay H.C., \& Ramos L. (1993). Response perseveration, inhibitory control, and central 
dopaminergic activity in childhood behavior disorders. Journal of Genetic Psychology, 154, 177 -188.

Dawson, G., Osterling, J., Rinaldi, J., Carver, L., \& McPartland, J. (2001). Brief report: Recognition memory and stimulus-reward associations: Indirect support for the role of ventromedial prefrontal dysfunction in autism. Journal of Autism and Developmental Disorders, 31, 337-341. doi: 10.1023/A:1010751404865

Demurie, E., Roeyers, H., Baeyens, D., \& Sonuga-Barke, E. (2011). Common alterations in sensitivity to type but not amount of reward in ADHD and autism spectrum disorders. Journal of Child Psychology and Psychiatry, 52(11), 1164-1172. doi: 10.1111/j.1469-7610.2010.02374.x

Demurie, E., Roeyers, H., Baeyens, D., \& Sonuga-Barke, E. (2012). Temporal discounting of monetary rewards in children and adolescents with ADHD and autism spectrum disorders. Developmental Science, 15(6), 791-800. doi: 10.1111/j.1467-7687.2012.01178.x

Desman, C., Petermann, F., \& Hampel, P. (2008). Deficit in response inhibition in children with attentiondeficit/hyperactivity disorder (ADHD): Impact of motivation? Child Neuropsychology, 14(6), 483-503. doi: 10.1080/09297040701625831

Dichter, G.S., Richey, J.A., Rittenberg, A.M., Sabatino, A., \& Bodfish, J.W. (2012). Reward circuitry function in autism during face anticipation and outcomes. Journal of Autism and Developmental Disorders, 42(2), 147-160. doi: 10.1007/s10803-011-1221-1

Garretson, H.B., Fein, D., \& Waterhouse, L. (1990). Sustained attention in children with autism. Journal of Autism and Developmental Disorders, 20, 101-114. doi: 10.1007/BF02206860

Geurts, H.M., Luman, M., \& van Meel, C. (2008). What's in a game: The effect of social motivation on interference control in boys with ADHD and autism spectrum disorders. Journal of Child Psychology and Psychiatry, 49(8), 848-857. doi: 10.1111/j.1469-7610.2008.01916.x

Geurts, H.M., Verté, S., Oosterlaan, J., Roeyers, H., \& Sergeant, J.A. (2004). How specific are executive functioning deficits in attention deficit hyperactivity disorder and autism? Journal of Child Psychology and Psychiatry, 45(4), 836-854. doi:10.1111/j.1469-7610.2004.00276.x

Grégoire, J. (2005). L'évaluation clinique de l'intelligence de l'enfant. Théorie et pratique du WISC-III, troisième édition. Sprimont : Mardaga.

Haenlein, M., \& Caul, W.F. (1987). Attention deficit disorder with hyperactivity: A specific hypothesis of reward dysfunction. Journal of the American Academy of Child and Adolescent Psychiatry, 26, 356-362. doi: 10.1097/00004583-198705000-00014

Jennings, J.R., van der Molen, M.W., Pelham, W., Debski, K.B., \& Hoza, B. (1997). Inhibition in boys with 
attention deficit hyperactivity disorder as indexed by heart rate change. Developmental Psychology, 33(2), 308-318. doi: 10.1037/0012-1649.33.2.308

Johnson, K.A., Wiersema, J.R., \& Kuntsi, J. (2009). What would Karl Popper say? Are current psychological theories of ADHD falsifiable? Behavioral and Brain Functions, 5, 15. doi: 10.1186/1744-9081-5-15

Johnson, M.W., \& Bickel, W.K. (2002). Within-subject comparison of real and hypothetical money rewards in delay discounting. Journal of the Experimental Analysis of Behavior, 77, 129-146. doi: 10.1901/jeab.2002.77-129

Karalunas, S.L., \& Huang-Pollock C.L. (2011). Examining relationships between executive functioning and delay aversion in attention deficit hyperactivity disorder. Journal of Clinical Child and Adolescent Psychology, 40, 837-847. doi: 10.1080/15374416.2011.614578

Knutson, B., Fong, G.W., Adams, C.M., Varner, J.L., \& Hommer, D. (2001). Dissociation of reward anticipation and outcome with event-related fMRI. Neuroreport, 12, 3683-3687. doi: 10.1097/00001756-20011204000016

Kohls, G., Herpertz-Dahlmann, B., \& Konrad, K. (2009). Hyperresponsiveness to social rewards in children and adolescents with attention-deficit/hyperactivity disorder (ADHD). Behavioral and Brain Functions, 5, 20. doi: $10.1186 / 1744-9081-5-20$

Kohls, G., Schulte-Rüther, M., Nehrkorn, B., Müller, K., Fink, G.R., Kamp-Becker, I., ... Konrad, K. (2012). Reward system dysfunction in autism spectrum disorders. Social Cognitive and Affective Neuroscience, Epub ahead of print.

Konrad, K., Gauggel, S., Manz, A., \& Schöll, M. (2000). Lack of inhibition: A motivational deficit in children with attention deficit/hyperactivity disorder and children with traumatic brain injury. Child Neuropsychology, 6(4), 286-296. doi: 10.1076/chin.6.4.286.3145

Krebs, R.M., Boehler, C.N., Roberts, K.C., Song, A.W., \& Woldorff, M.G. (2012). The involvement of the dopaminergic midbrain and cortico-striatal-thalamic circuits in the integration of reward prospect and task demands. Cerebral Cortex, 22(3), 607-615.

Leotti, L.A., \& Wager, T.D. (2010). Motivational influences on response inhibition measures. Journal of Experimental Psychology - Human Perception and Performance, 36(2), 430-447. doi: 10.1037/a0016802

Lijffijt, M., Kenemans, J.L., Verbaten, M.N., \& van Engeland, H. (2005). A meta-analytic review of stopping performance in attention-deficit/hyperactivity disorder: Deficient inhibitory motor control? Journal of Abnormal Psychology, 114(2), 216-222. doi: 10.1037/0021-843X.114.2.216 
Logan, G. D. (1994). On the ability to inhibit thought or action: A users' guide to the stop signal paradigm. In D. Dagenbach \& T. H. Carr (Eds.), Inhibitory processes in attention, memory, and learning (pp.189-239). San Diego, CA: Academic Press.

Lord, C., Risi, S., Lambrecht, L., Cook, E.H., Leventhal, B.L., DiLavore, P.C., ... Rutter M. (2004). The Autism Diagnostic Observation Schedule-Generic: A standard measure of social and communication deficits associated with the spectrum of autism. Journal of Autism and Developmental Disorders, 30, 205-223. doi: 10.1023/A:1005592401947

Lord, C., Rutter, M., \& Lecouteur, A. (1994). Autism Diagnostic Interview-Revised: A revised version of a diagnostic interview for caregivers of individuals with possible pervasive developmental disorders. Journal of Autism and Developmental Disorders, 24, 659-685. doi: 10.1007/BF02172145

Luman, M., Oosterlaan, J., \& Sergeant, J.A. (2005). The impact of reinforcement contingencies on AD/HD: A review and theoretical appraisal. Clinical Psychology Review, 25, 183-213. doi: 10.1016/j.cpr.2004.11.001

Luman, M., Tripp, G., \& Scheres, A. (2010). Identifying the neurobiology of altered reinforcement sensitivity in ADHD: A review and research agenda. Neuroscience and Biobehavioral Reviews, 34(5), 744-754. doi: 10.1016/j.neubiorev.2009.11.021

Mischel, W., Ayduk, O., Berman, M.G., Casey, B.J., Gotlib, I.H., Jonides, J., ... Shoda, Y. (2011). 'Willpower' over the life span: Decomposing self-regulation. Social Cognitive and Affective Neuroscience, 6(2), 252256. doi: $10.1093 / \mathrm{scan} / \mathrm{nsq} 081$

Myerson, J., Green, L., \& Warusawitharana, M. (2001). Area under curve as a measure of discounting. Journal of the Experimental Analysis of Behaviour, 76, 235-243. doi: 10.1901/jeab.2001.76-235

Nigg, J.T. (2000). On inhibition/disinhibition in developmental psychopathology: Views from cognitive and personality psychology and a working inhibition taxonomy. Psychological Bulletin, 126(2), 220-246. doi: $10.1037 / / 0033-2909.126 .2 .220$

Nigg, J.T. (2001). Is ADHD a disinhibitory disorder? Psychological Bulletin, 127(5), 571-598. doi: $10.1037 / / 0033-2909.127 .5 .571$

Oosterlaan, J., \& Sergeant, J.A. (1998). Effects of reward and response cost on response inhibition in AD/HD, disruptive, anxious, and normal children. Journal of Abnormal Child Psychology, 26(3), 161-174. doi: 10.1023/A:1022650216978 
Oosterlaan, J., Logan, G.D., \& Sergeant, J.A. (1998). Response inhibition in AD/HD, CD, comorbid $\mathrm{AD} / \mathrm{HD}+\mathrm{CD}$, anxious, and control children: A meta-analysis of studies with the stop task. Journal of Child Psychology and Psychiatry, 39(3), 411-425. doi: 10.1017/S0021963097002072

Ozonoff, S., \& Jensen, J. (1999). Brief report: Specific executive function profiles in three neurodevelopmental disorders. Journal of Autism and Developmental Disorders, 29(2), 171-177. doi: 10.1023/A:1023052913110

Parry, P.A., \& Douglas, V.I. (1983). Effects of reinforcement on concept-identification in hyperactive-children. Journal of Abnormal Child Psychology, 11(2), 327-340. doi: 10.1007/BF00912095

Pelham, W.E., Gnagy, E.M., Greenslade, K.E., \& Milich, R. (1992). Teacher ratings of DSM-III-R symptoms for disruptive behavior disorders. Journal of the American Academy of Child and Adolescent Psychiatry, 31, 210-218. doi: 10.1097/00004583-199203000-00006

Rademacher, L., Krach, S., Kohls, G., Irmak, A., Gründer, G., \& Spreckelmeyer, K.N. (2010). Dissociation of neural networks for anticipation and consumption of monetary and social rewards. NeuroImage, 49(4), 3276-3285. doi: 10.1016/j.neuroimage.2009.10.089

Raymaekers, R., Antrop, I., van der Meere, J., Wiersema, J.R., \& Roeyers, H. (2007). HFA and ADHD: A direct comparison on state regulation and response inhibition. Journal of Clinical and Experimental Neuropsychology, 29(4), 418-427. doi: 10.1080/13803390600737990

Rommelse, N.N.J., Altink, M.E., de Sonneville, L.M.J., Buschgens, C.J.M., Buitelaar, J., Oosterlaan, J., \& Sergeant, J.A. (2007). Are motor inhibition and cognitive flexibility dead ends in ADHD? Journal of Abnormal Child Psychology, 35, 957-967. doi: 10.1007/s10802-007-9146-z

Rutter, M., Bailey, A., \& Lord, C. (2003). Social Communication Questionnaire. California: Western Psychological Services.

Sagvolden, T., Aase, H., Zeiner, P., \& Berger, D. (1998). Altered reinforcement mechanisms in attentiondeficit/hyperactivity disorder. Behavioural Brain Research, 94, 1-10. doi: 10.1016/S01664328(97)00170-8

Sagvolden, T., Johansen, E.B., Aase, H., \& Russell, V.A. (2005). A dynamic developmental theory of attentiondeficit/hyperactivity disorder (ADHD) predominantly hyperactive/impulsive and combined subtypes. Behavioral and Brain Sciences, 28(3), 397-419. doi: 10.1017/S0140525X05000075

Sanders, A.F. (1983). Towards a model of stress and human performance. Acta Psychologica, 53, 61-97. doi: $\underline{10.1016 / 0001-6918(83) 90016-1}$ 
Scheres, A., Dijkstra, M., Ainslie, E., Balkan, J., Reynolds, B., Sonuga-Barke, E., \& Castellanos, F.X. (2006). Temporal and probabilistic discounting of rewards in children and adolescents: Effects of age and ADHD symptoms. Neuropsychologia, 44(11), 2092-2103. doi: 10.1016/j.neuropsychologia.2005.10.012

Scheres, A., Milham, M.P., Knutson, B., \& Castellanos, F.X. (2007). Ventral striatal hyporesponsiveness during reward anticipation in attention-deficit/hyperactivity disorder. Biological Psychiatry, 61, 720-724. doi: 10.1016/j.biopsych.2006.04.042

Scheres, A., Oosterlaan, J., \& Sergeant, J.A. (2001). Response inhibition in children with DSM-IV subtypes of AD/HD and related disruptive disorders: The role of reward. Child Neuropsychology, 7(3), 172-189. doi: 10.1076/chin.7.3.172.8746

Schopler, E., Reichler, R.J., Devellis, R.F., \& Daly, K. (1980). Toward objective classification of childhood autism: Childhood autism rating-scale (CARS). Journal of Autism and Developmental Disorders, 10, 91103. doi: $10.1007 / \mathrm{BF} 02408436$

Scheres, A., Sumiya, M., \& Thoeny, A. L. (2010). Studying the relation between temporal reward discounting tasks used in populations with ADHD: A factor analysis. International Journal of Methods in Psychiatric Research, 19(3), 167-176. doi: 10.1002/mpr.309

Schultz, R.T. (2005). Developmental deficits in social perception in autism: The role of the amygdala and fusiform face area. International Journal of Developmental Neuroscience, 23, 125-141. doi: 10.1016/j.ijdevneu.2004.12.012

Scott-Van Zeeland, A.A., Dapretto, M., Ghahremani, D.G., Poldrack, R.A., \& Bookheimer, S.Y. (2010). Reward processing in autism. Autism Research, 3, 1-15. doi: 10.1002/aur.122

Sergeant, J. (2000). The cognitive-energetic model: An empirical approach to attention-deficit hyperactivity disorder. Neuroscience and Biobehavioral Reviews, 24, 7-12. doi: 10.1016/S0149-7634(99)00060-3

Sergeant, J.A., Oosterlaan, J., \& Van der Meere, J.J. (1999). Information processing in attentiondeficit/hyperactivity disorder. In H.C. Quay, \& A.E. Hogan (Eds.), Handbook of disruptive behavior disorders (pp. 75-104). New York: Plenum.

Shaffer, D., Fisher, P., Lucas, C.P., Dulcan, M.K., \& Schwab-Stone, M.E. (2000). NIMH Diagnostic Interview Schedule for Children Version IV: Description, differences from previous versions, and reliability of some common diagnoses. Journal of the American Academy of Child and Adolescent Psychiatry, 39, 2838. doi: 10.1097/00004583-200001000-00014 
Shanahan, M.A., Pennington, B.R., \& Willcutt, E.W. (2008). Do motivational incentives reduce the inhibition deficit in ADHD? Developmental Neuropsychology, 33(2), 137-159. doi: 10.1080/87565640701884238

Simmonds, D.J., Pekar, J.J., \& Mostofsky, S.H. (2008). Meta-analysis of go/no-go tasks demonstrating that fMRI activation associated with response inhibition is task-dependent. Neuropsychologia, 46, 224-232. doi: 10.1016/j.neuropsychologia.2007.07.015

Slusarek, M., Velling, S., Bunk, D., \& Eggers, C. (2001). Motivational effects on inhibitory control in children with ADHD. Journal of the American Academy of Child and Adolescent Psychiatry, 40(3), 355-363. doi: $10.1097 / 00004583-200103000-00016$

Solanto, M.V., Abikoff, H., Sonuga-Barke, E., Schachar, R., Logan, G.D., Wigal, T., ... Turkel, E. (2001). The ecological validity of delay aversion and response inhibition as measures of impulsivity in AD/HD: A supplement to the NIMH multimodal treatment study of AD/HD. Journal of Abnormal Child Psychology, 29(3), 215-228. doi: 10.1023/A:1010329714819

Sonuga-Barke, E.J.S., \& Fairchild, G. (2012). Neuroeconomics of attention-deficit/hyperactivity disorder: Differential influences of medial, dorsal and ventral prefrontal brain networks on sub-optimal decisionmaking? Biological Psychiatry, epub ahead of print. doi: 10.1016/j.biopsych.2012.04.004

Sonuga-Barke, E.J.S., Taylor, E., Sembi, S., \& Smith, J. (1992). Hyperactivity and delay aversion: I. The effect of delay on choice. Journal of Child Psychology and Psychiatry and Allied Disciplines, 33(2), 387-398. doi: 10.1111/j.1469-7610.1992.tb00874.x

Spreckelmeyer, K.N., Krach, S., Kohls, G., Rademacher, L., Irmak, A., Konrad, K., ... Gründer, G. (2009). Anticipation of monetary and social reward differently activates mesolimbic brain structures in men and women. Scan, 4, 158-165. doi: 10.1093/scan/nsn051

Ströhle, A., Stoy, M., Wrase, J., Schwarzer, S., Schlagenhauf, F., Huss, M., ... Heinz, A. (2007). Reward anticipation and outcomes in adult males with attention-deficit/hyperactivity disorder. NeuroImage, 39, 966-972. doi: 10.1016/j.neuroimage.2007.09.044

Tripp, G., \& Alsop, B. (1999). Sensitivity to reward frequency in boys with attention deficit hyperactivity disorder. Journal of Clinical Child Psychology, 28(3), 366-375. doi: 10.1207/S15374424jccp280309

Van De Voorde, S., Roeyers, H., Verté, S., \& Wiersema, J.R. (2010). Working memory, response inhibition, and within-subject variability in children with attention-deficit/hyperactivity disorder or reading disorder. Journal of Clinical and Experimental Neuropsychology, 32, 366-379. doi: 10.1080/13803390903066865 
Verté, S., Geurts, H.M., Roeyers, H., Oosterlaan, J., \& Sergeant, J.A. (2005). Executive functioning in children with autism and Tourette syndrome. Development and Psychopathology, 17(2), 415-445. doi: $10.1017 / \mathrm{S} 0954579405050200$

Wechsler, D. (1991). Wechsler Intelligence Scale for Children (3rd ed.). New York: Psychological Corporation.

Wodka, E.L., Mahone, E. M., Blankner, J.G., Larson, J.C.G., Fotedar, S., Denckla, M.B., \& Mostofsky, S.H. (2007). Evidence that response inhibition is a primary deficit in ADHD. Journal of Clinical and Experimental Neuropsychology, 29(4), 345-356. doi: 10.1080/13803390600678046 
Table 1 Descriptive characteristics of the sample

\begin{tabular}{|c|c|c|c|c|c|c|c|c|c|}
\hline & \multicolumn{2}{|c|}{$\begin{array}{c}\text { Controls } \\
\mathrm{n}=41\end{array}$} & \multicolumn{2}{|c|}{$\begin{array}{c}\text { ADHD } \\
\mathrm{n}=34\end{array}$} & \multicolumn{2}{|c|}{$\begin{array}{l}\text { ASD } \\
\mathrm{n}=36\end{array}$} & \multicolumn{2}{|c|}{$\begin{array}{l}\text { ADHD+ASD } \\
\quad \mathrm{n}=16\end{array}$} & \\
\hline \multirow[t]{2}{*}{$\begin{array}{l}\text { Sex } \\
\text { Male } \\
\text { Female } \\
\end{array}$} & \multicolumn{2}{|c|}{$\begin{array}{l}30 \\
11 \\
\end{array}$} & \multicolumn{2}{|c|}{$\begin{array}{c}28 \\
6 \\
\end{array}$} & \multicolumn{2}{|c|}{$\begin{array}{c}33 \\
3\end{array}$} & \multicolumn{2}{|c|}{$\begin{array}{c}13 \\
3 \\
\end{array}$} & \multirow[t]{2}{*}{$\chi^{2}(3)=4.43, p=.22$} \\
\hline & M & SD & $\mathbf{M}$ & $\mathbf{M}$ & SD & SD & $\mathbf{M}$ & SD & \\
\hline Age in months & 137.39 & 22.23 & 137.41 & 22.14 & 136.42 & 26.31 & 131.56 & 28.10 & $\mathrm{~F}(3,123)=.26, \mathrm{p}=.86$ \\
\hline Estimated FSIQ & 105.95 & 8.84 & 101.76 & 11.62 & 105.08 & 12.96 & 99.13 & 11.07 & $F(3,123)=1.98, p=.12$ \\
\hline SCQ & 4.10 & 3.12 & 5.71 & 3.00 & 13.58 & 5.56 & 17.63 & 5.98 & $\begin{array}{l}\mathrm{F}(3,123)=58.70, \mathrm{p}<.001 \\
\text { Controls }=\mathrm{ADHD}<\mathrm{ASD}<\mathrm{ADHD}+\mathrm{ASD}\end{array}$ \\
\hline \multicolumn{10}{|l|}{ Parent DBDRS } \\
\hline IA & 4.32 & 3.27 & 15.29 & 5.72 & 13.31 & 6.13 & 14.13 & 5.75 & $\begin{array}{l}\mathrm{F}(3,123)=34.63, \mathrm{p}<.001 \\
\text { Controls }<\mathrm{ADHD}=\mathrm{ASD}=\mathrm{ADHD}+\mathrm{ASD}\end{array}$ \\
\hline H/I & 2.34 & 2.72 & 12.99 & 5.24 & 9.53 & 4.87 & 13.44 & 6.41 & $\begin{array}{l}\mathrm{F}(3123)=41.31, \mathrm{p}<.001 \\
\text { Controls }<\mathrm{ASD}<\mathrm{ADHD}=\mathrm{ADHD}+\mathrm{ASD}\end{array}$ \\
\hline \multicolumn{10}{|l|}{ Teacher DBDRS } \\
\hline IA & 2.86 & 3.39 & 10.23 & 6.11 & 8.41 & 6.38 & 10.44 & 6.11 & $\begin{array}{l}\mathrm{F}(3,102)=12.63, \mathrm{p}<.001 \\
\text { Controls }<\mathrm{ADHD}=\mathrm{ASD}=\mathrm{ADHD}+\mathrm{ASD}\end{array}$ \\
\hline $\mathbf{H} / \mathbf{I}$ & 1.69 & 2.67 & 8.53 & 5.79 & 4.45 & 5.21 & 7.42 & 6.08 & $\begin{array}{l}\mathrm{F}(3,102)=11.80, \mathrm{p}<.001 \\
\text { Controls }=\mathrm{ASD}<\mathrm{ADHD} ; \mathrm{ADHD}= \\
\mathrm{ADHD}+\mathrm{ASD} ; \mathrm{ASD}=\mathrm{ADHD}+\mathrm{ASD}\end{array}$ \\
\hline
\end{tabular}

Note: FSIQ: full scale intelligence quotient, SCQ: Social Communication Questionnaire, DBDRS: Disruptive Behavior Disorder rating scale, IA: inattention subscale scores, H/I: hyperactive/impulsive subscale scores 


\section{For the 75-25 monetary go/no-go task}

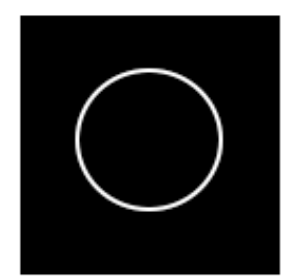

no reward

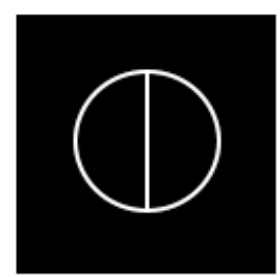

medium reward

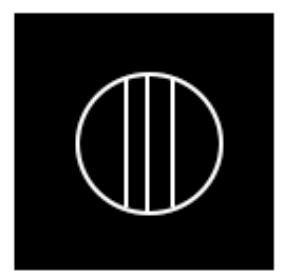

high reward

$$
\begin{aligned}
& \text { No reward cue: } \quad \text { - go: } \quad \text { - reaction: }+0 \\
& \text { - no reaction: } 0 \\
& \text { - reaction: } 0 \\
& \text { - no reaction: }+0
\end{aligned}
$$

Medium reward cue: - go: $\quad$ - reaction: +4 eurocent

- no reaction: 0

- no-go: $\quad$ - reaction: 0

- no reaction: +12 eurocent

High reward cue: $\quad$ - go: $\quad$ - reaction: +10 eurocent

- no reaction: 0

- no-go: $\quad$ - reaction: 0

- no reaction: +30 eurocent

Figure 1. Reward cues in the 75-25 monetary task

\section{Go-trial in the MID tasks:}

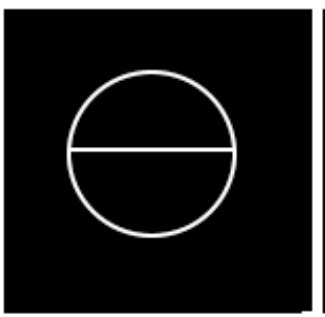

$500 \mathrm{~ms}$

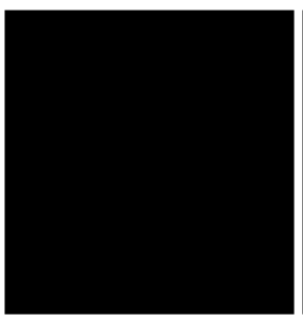

$2000-2500 \mathrm{~ms}$

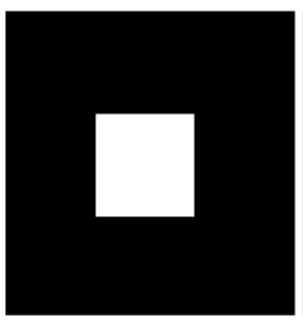

$260 \mathrm{~ms}$ (response window: $500 \mathrm{~ms}$ )

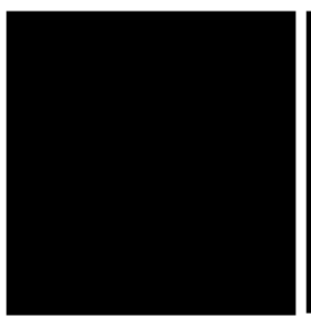

$1000-1500 \mathrm{~ms}$

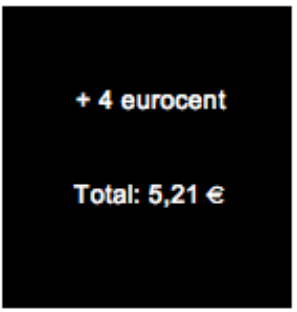

$1740 \mathrm{~ms}$

No-go trial in the SID tasks:

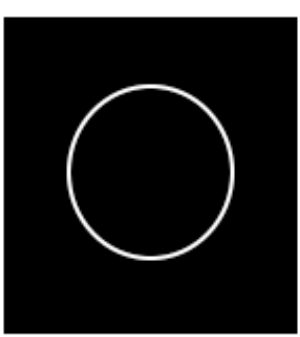

$500 \mathrm{~ms}$

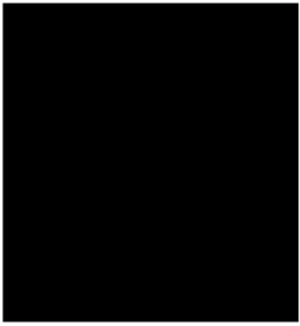

$2000-2500 \mathrm{~ms}$

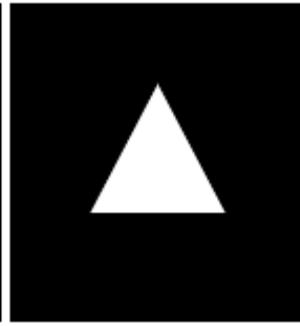

$260 \mathrm{~ms}$ (response window: $500 \mathrm{~ms}$ )

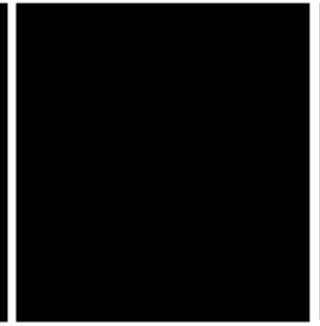

$1000-1500 \mathrm{~ms}$

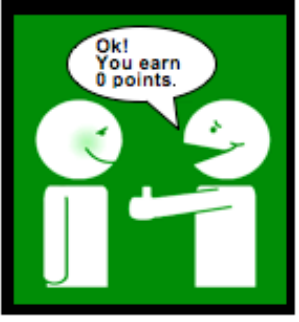

$1740 \mathrm{~ms}$

Figure 2. A trial of the monetary and social tasks 


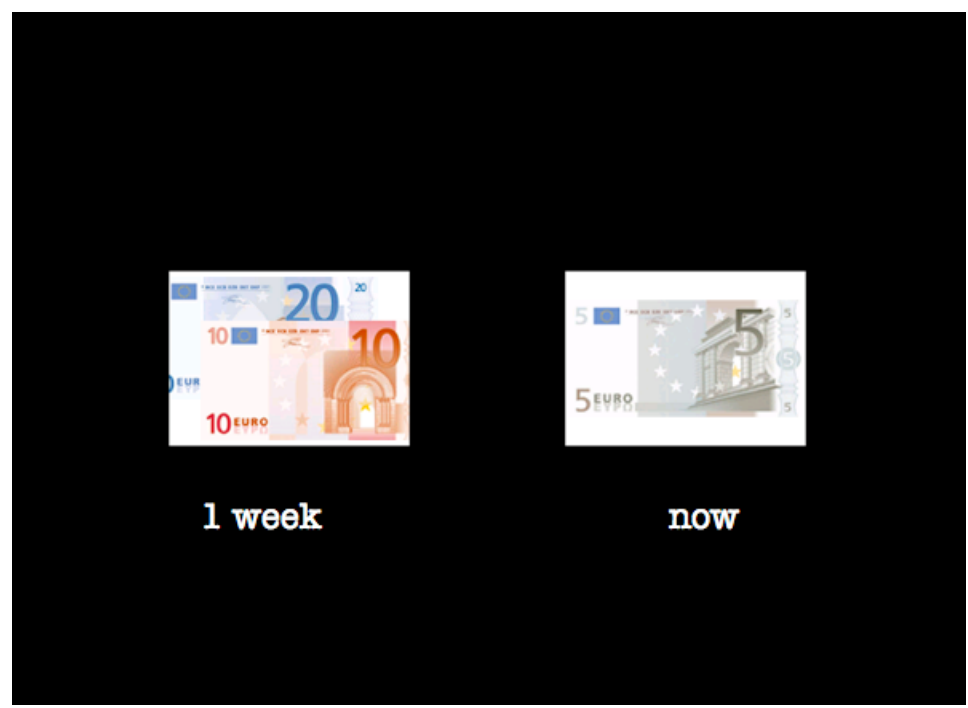

Figure 3. Trial of the monetary temporal discounting task. Instruction: Which reward do you prefer, taking the delay into account?

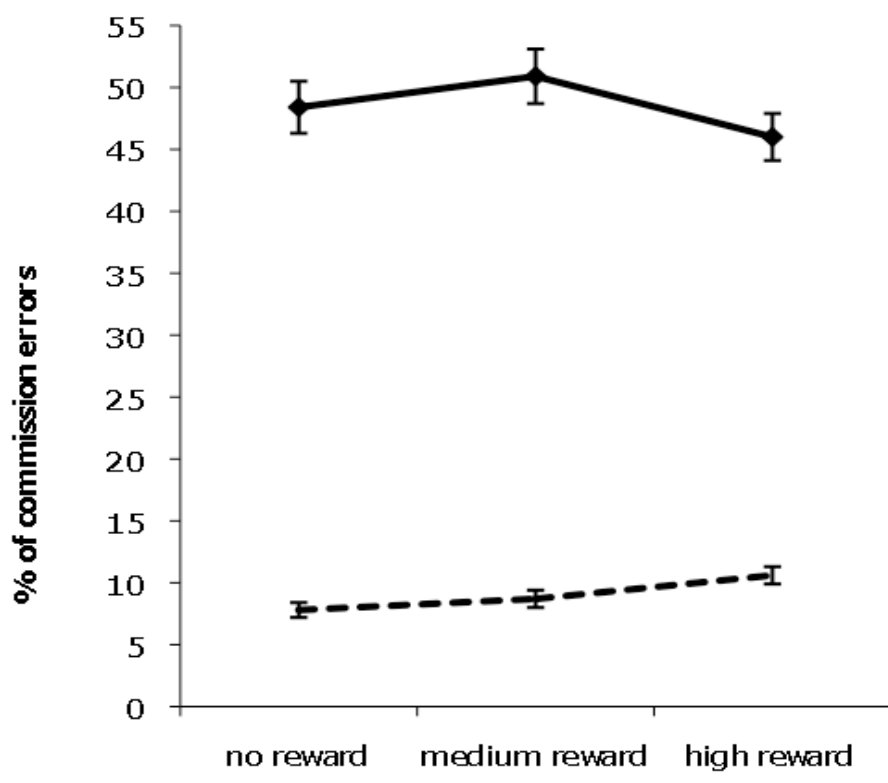

Figure 4. Interaction between inhibitory load and reward amount for commission errors (error bars refer to standard errors) 


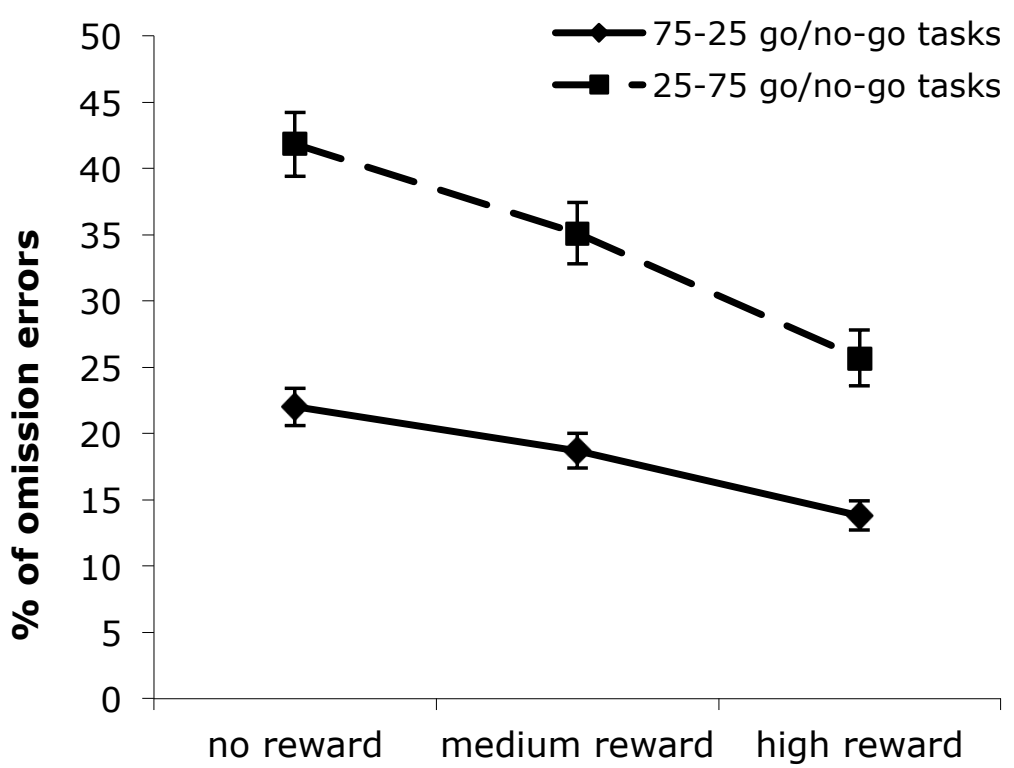

Figure 5. Interaction between inhibitory load and reward amount for omission errors (error bars refer to standard errors)

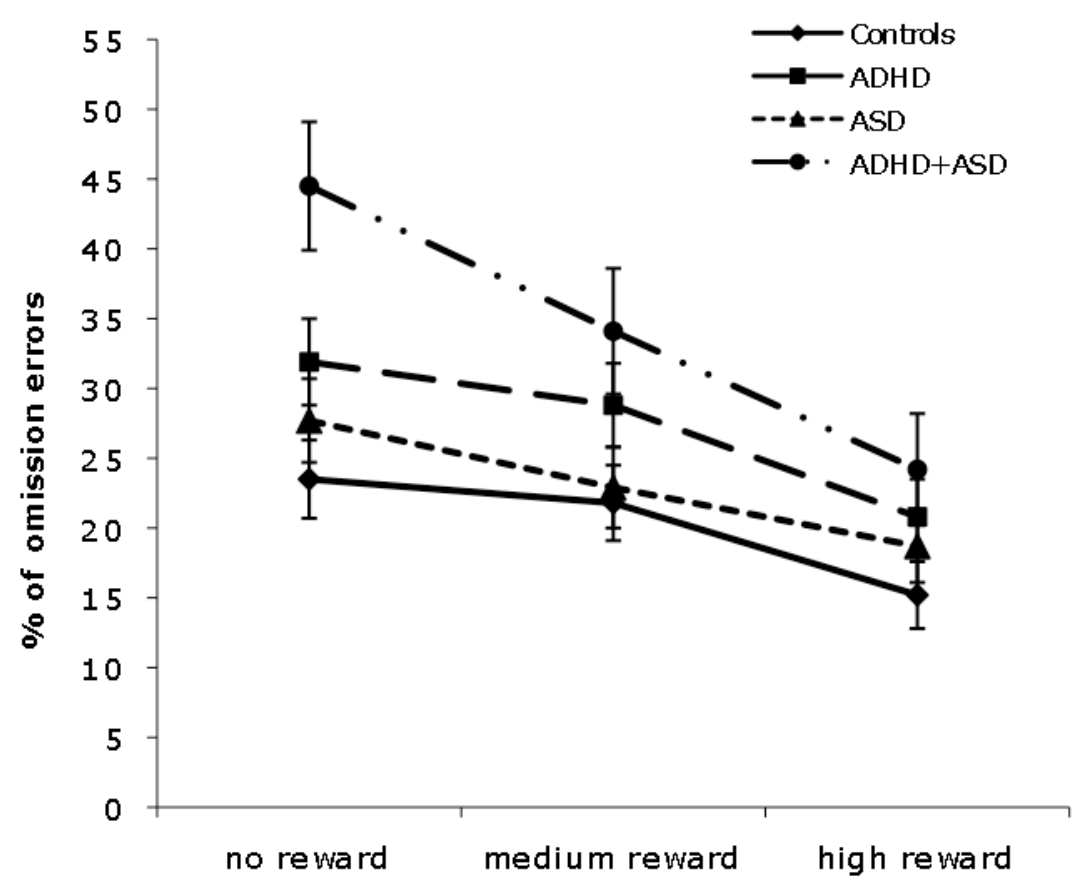

Figure 6. Interaction between group and reward intensity for omission errors (error bars refer to standard errors) 
Table S1. Group means and standard deviations per condition for the four dependent variables

\begin{tabular}{|c|c|c|c|c|c|c|c|c|c|c|c|c|c|c|c|c|}
\hline \multirow{3}{*}{$\begin{array}{l}\text { High inhibitory } \\
\text { load }\end{array}$} & \multicolumn{8}{|c|}{ MID task } & \multicolumn{8}{|c|}{ SID task } \\
\hline & \multicolumn{2}{|c|}{ Controls } & \multicolumn{2}{|c|}{ ADHD } & \multicolumn{2}{|c|}{ ASD } & \multicolumn{2}{|c|}{$A D H D+A S D$} & \multicolumn{2}{|c|}{ Controls } & \multicolumn{2}{|c|}{ ADHD } & \multicolumn{2}{|c|}{ ASD } & \multicolumn{2}{|c|}{ ADHD+ASD } \\
\hline & $M$ & SD & $M$ & SD & M & SD & M & SD & M & SD & M & SD & M & SD & $M$ & SD \\
\hline \multicolumn{17}{|c|}{ Commission errors } \\
\hline 0 eurocent & 0,45 & 0,27 & 0,45 & 0,25 & 0,49 & 0,29 & 0,56 & 0,26 & 0,46 & 0,25 & 0,43 & 0,23 & 0,50 & 0,28 & 0,51 & 0,20 \\
\hline 12 eurocent & 0,47 & 0,29 & 0,47 & 0,24 & 0,49 & 0,27 & 0,57 & 0,22 & 0,50 & 0,24 & 0,55 & 0,25 & 0,49 & 0,29 & 0,53 & 0,22 \\
\hline 30 eurocent & 0,41 & 0,24 & 0,44 & 0,24 & 0,41 & 0,24 & 0,51 & 0,24 & 0,46 & 0,21 & 0,46 & 0,20 & 0,46 & 0,28 & 0,53 & 0,15 \\
\hline \multicolumn{17}{|l|}{ Omission errors } \\
\hline 0 eurocent & 0,15 & 0,16 & 0,22 & 0,16 & 0,19 & 0,15 & 0,32 & 0,17 & 0,15 & 0,13 & 0,22 & 0,17 & 0,18 & 0,15 & 0,33 & 0,16 \\
\hline 4 eurocent & 0,14 & 0,16 & 0,18 & 0,16 & 0,14 & 0,14 & 0,18 & 0,17 & 0,17 & 0,11 & 0,22 & 0,15 & 0,17 & 0,15 & 0,30 & 0,20 \\
\hline 10 eurocent & 0,10 & 0,10 & 0,15 & 0,17 & 0,11 & 0,11 & 0,14 & 0,13 & 0,13 & 0,11 & 0,16 & 0,13 & 0,14 & 0,14 & 0,18 & 0,16 \\
\hline \multicolumn{17}{|l|}{ RT } \\
\hline 0 eurocent & 330,37 & 32,21 & 339,81 & 32,89 & 331,52 & 34,60 & 330,84 & 34,55 & 337,25 & 31,41 & 337,47 & 34,42 & 323,90 & 32,92 & 346,86 & 37,66 \\
\hline 4 eurocent & 333,09 & 34,29 & 340,79 & 36,10 & 329,61 & 37,41 & 329,93 & 38,56 & 336,94 & 34,47 & 335,05 & 31,72 & 327,23 & 34,70 & 351,84 & 33,79 \\
\hline 10 eurocent & 332,46 & 30,47 & 329,99 & 34,48 & 330,71 & 41,15 & 324,95 & 33,81 & 330,04 & 32,55 & 327,09 & 32,06 & 324,77 & 37,51 & 337,53 & 32,05 \\
\hline \multicolumn{17}{|l|}{ SD-RT } \\
\hline 0 eurocent & 63,63 & 10,91 & 72,39 & 10,83 & 67,29 & 14,40 & 72,06 & 9,54 & 68,60 & 10,46 & 73,16 & 12,85 & 70,90 & 17.00 & 72.00 & 13,49 \\
\hline 4 eurocent & 65,50 & 12,55 & 74,55 & 12,21 & 62,04 & 14,17 & 69,75 & 15,41 & 69,13 & 14,50 & 74,13 & 9,94 & 66,66 & 13,80 & 70,26 & 13,84 \\
\hline 10 eurocent & 65,76 & 11,50 & 68,32 & 10,99 & 62,56 & 8,94 & 66,25 & 16,28 & 67,65 & 12,65 & 70,80 & 13,70 & 64,92 & 14,02 & 71,55 & 12,88 \\
\hline & \multicolumn{2}{|c|}{ Controls } & \multicolumn{2}{|c|}{ ADHD } & \multicolumn{2}{|c|}{ ASD } & \multicolumn{2}{|c|}{ ADHD+ASD } & \multicolumn{2}{|c|}{ Controls } & \multicolumn{2}{|c|}{ ADHD } & \multicolumn{2}{|c|}{ ASD } & \multicolumn{2}{|c|}{ ADHD+ASD } \\
\hline load & M & SD & M & SD & M & SD & M & SD & $\mathrm{M}$ & SD & $M$ & SD & M & SD & $M$ & SD \\
\hline \multicolumn{17}{|c|}{ Commission errors } \\
\hline 0 eurocent & 0,06 & 0,08 & 0,07 & 0,07 & 0,06 & 0,06 & 0,11 & 0,08 & 0,07 & 0,06 & 0,08 & 0,08 & 0,07 & 0,07 & 0,12 & 0,17 \\
\hline 4 eurocent & 0,08 & 0,08 & 0,08 & 0,07 & 0,08 & 0,08 & 0,12 & 0,09 & 0,07 & 0,07 & 0,09 & 0,09 & 0,08 & 0,10 & 0,10 & 0,09 \\
\hline 10 eurocent & 0,08 & 0,09 & 0,09 & 0,07 & 0,11 & 0,10 & 0,15 & 0,09 & 0,08 & 0,08 & 0,09 & 0,06 & 0,09 & 0,08 & 0,17 & 0,11 \\
\hline
\end{tabular}

\section{Omission errors}




\begin{tabular}{|c|c|c|c|c|c|c|c|c|c|c|c|c|c|c|c|c|}
\hline 0 eurocent & 0,31 & 0,27 & 0,38 & 0,28 & 0,36 & 0,30 & 0,54 & 0,32 & 0,33 & 0,27 & 0,45 & 0,26 & 0,38 & 0,27 & 0,59 & 0,23 \\
\hline 12 eurocent & 0,27 & 0,24 & 0,35 & 0,30 & 0,26 & 0,24 & 0,41 & 0,25 & 0,29 & 0,27 & 0,40 & 0,26 & 0,35 & 0,27 & 0,48 & 0,25 \\
\hline 30 eurocent & 0,19 & 0,20 & 0,22 & 0,25 & 0,21 & 0,26 & 0,24 & 0,26 & 0,20 & 0,23 & 0,30 & 0,24 & 0,29 & 0,26 & 0,41 & 0,27 \\
\hline \multicolumn{17}{|l|}{ RT } \\
\hline 0 eurocent & 384,43 & 46,15 & 376,60 & 35,42 & 371,18 & 47,03 & 378,15 & 44,86 & 372,53 & 42,76 & 394,78 & 52,53 & 383,68 & 42,20 & 370,81 & 42,69 \\
\hline 12 eurocent & 375,70 & 39,97 & 365,47 & 39,86 & 361.00 & 36,14 & 359,65 & 38,76 & 368,52 & 41,52 & 368,88 & 36,23 & 362,77 & 45,79 & 363,09 & 44,24 \\
\hline 30 eurocent & 347,07 & 44,55 & 345,19 & 44,22 & 339,66 & 50,10 & 332,18 & 35,85 & 348,23 & 43,37 & 359,21 & 42,03 & 354,09 & 46,26 & 363,18 & 46,02 \\
\hline \multicolumn{17}{|l|}{ SD-RT } \\
\hline 0 eurocent & 57,41 & 19,31 & 61,95 & 20,86 & 60,71 & 22,71 & 74,01 & 28,38 & 64,68 & 18,54 & 54,19 & 23,96 & 63,61 & 18,46 & 62,28 & 39,05 \\
\hline 12 eurocent & 65,74 & 18,14 & 71,46 & 27,92 & 63,76 & 19,42 & 76,23 & 16,91 & 62,67 & 21,65 & 66,24 & 16,01 & 62,80 & 17,84 & 64,44 & 18,16 \\
\hline 30 eurocent & 62,35 & 17,43 & 60,95 & 18,94 & 62,67 & 21,74 & 62,97 & 26,63 & 62.00 & 18,38 & 64,81 & 21,62 & 60,91 & 17,09 & 60,67 & 32,54 \\
\hline
\end{tabular}

